\title{
On symplectic polar spaces over non-perfect fields of characteristic 2
}

\author{
B. De Bruyn* and A. Pasini
}

July 20, 2009

\begin{abstract}
Given a field $\mathbb{K}$ of characteristic 2 and an integer $n \geq 2$, let $W(2 n-$ $1, \mathbb{K})$ be the symplectic polar space defined in $\operatorname{PG}(2 n-1, \mathbb{K})$ by a nondegenerate alternating form of $V(2 n, \mathbb{K})$ and let $Q(2 n, \mathbb{K})$ be the quadric of $\mathrm{PG}(2 n, \mathbb{K})$ associated to a non-singular quadratic form of Witt index $n$. In the literature it is often claimed that $W(2 n-1, \mathbb{K}) \cong Q(2 n, \mathbb{K})$. This is true when $\mathbb{K}$ is perfect, but false otherwise. In this paper we modify the previous claim in order to obtain a statement that is correct for any field of characteristic 2. Explicitly, we prove that $W(2 n-1, \mathbb{K})$ is indeed isomorphic to a non-singular quadric $Q$, but when $\mathbb{K}$ is nonperfect the nucleus of $Q$ has vector dimension greater than 1 . So, in this case, $Q(2 n, \mathbb{K})$ is a proper subgeometry of $W(2 n-1, \mathbb{K})$. We show that, in spite of this fact, $W(2 n-1, \mathbb{K})$ can be embedded in $Q(2 n, \mathbb{K})$ as a subgeometry and that this embedding induces a full embedding of the dual $D W(2 n-1, \mathbb{K})$ of $W(2 n-1, \mathbb{K})$ into the dual $D Q(2 n, \mathbb{K})$ of $Q(2 n, \mathbb{K})$.
\end{abstract}

\section{Introduction}

Given a field $\mathbb{K}$ of characteristic 2 and an integer $n \geq 2$, let $W(2 n-1, \mathbb{K})$ be the symplectic polar space defined in $\operatorname{PG}(2 n-1, \mathbb{K})$ by a non-degenerate alternating form of $V(2 n, \mathbb{K})$ and $Q(2 n, \mathbb{K})$ be the quadric of $\mathrm{PG}(2 n, \mathbb{K})$ associated to a nonsingular quadratic form of Witt index $n$. The following claim is often met in the literature:

$(\dagger) W(2 n-1, \mathbb{K}) \cong Q(2 n, \mathbb{K})$.

However, $(\dagger)$ is false if stated in this general form. Indeed, $(\dagger)$ is true if $\mathbb{K}$ is perfect (in particular, finite), but false otherwise. In the case $n=2$, this fact is pointed out by Van Maldeghem [6, 3.4.13]. In this note we shall discuss the general case, also giving more information than in $[6,3.4 .13]$.

We firstly state some notation. We denote by $\operatorname{rk}_{\text {gen }}(W(2 n-1, \mathbb{K}))$ the generating rank of $W(2 n-1, \mathbb{K})$, namely the minimal size of a spanning set of

*Postdoctoral Fellow of the Research Foundation - Flanders 
points of $W(2 n-1, \mathbb{K})$. The embedding rank of $W(2 n-1, \mathbb{K})$, namely the vector dimension of the universal full projective embedding of $W(2 n-1, \mathbb{K})$ (which is well known to exist), will be denoted by $\operatorname{rk}_{\mathrm{emb}}(W(2 n-1, \mathbb{K}))$. We denote by $\mathbb{K}^{2}$ the subfield of $\mathbb{K}$ consisting of all square elements of $\mathbb{K}$ and by $d$ the degree of $\mathbb{K}$ over $\mathbb{K}^{2}$. Note that $d$ might be infinite. When $d$ is infinite we denote by $\delta$ the least ordinal number of cardinality equal to $d$. When $d$ is finite, we set $\delta=d$. Given a basis $\Lambda=\left\{\lambda_{i}\right\}_{0 \leq i<\delta}$ of $\mathbb{K}$ over $\mathbb{K}^{2}$, where $\lambda_{0} \in \mathbb{K}^{2}$ (for instance, $\lambda_{0}=1$ ), we denote by $Q_{\Lambda}(2 n-1+d, \mathbb{K})$ the quadric of $\mathrm{PG}(2 n-1+d, \mathbb{K})$ associated to the following quadratic form, with respect to a basis of $V(2 n+d, \mathbb{K})$ ordered in accordance to $2 n+\delta$ :

$$
\text { (*) } q_{\Lambda}\left(\left(x_{i}\right)_{0 \leq i<2 n+\delta}\right):=\sum_{i=0}^{n-1} x_{i} x_{n+i}+\sum_{0 \leq i<\delta} \lambda_{i} x_{2 n+i}^{2} .
$$

The second sum of $(*)$ is well-defined even if $d$ is infinite, since only a finite number of non-null entries occur in the sequence $\left(x_{i}\right)_{0 \leq i<2 n+\delta}$. Note also that, when $i$ is an infinite ordinal number, then $k+i=i$ for every finite ordinal number $k$ (but $i<i+k$ if $k>0$ ). So, if $i$ is infinite then $2 n+i=i$, the integer $2 n$ being regarded as a finite ordinal number in this context. Clearly, if $\delta=1$ then $Q_{\Lambda}(2 n, \mathbb{K}) \cong Q(2 n, \mathbb{K})$. We can now state the main result of this paper, to be proved in Section 2 .

Theorem 1.1 We have $W(2 n-1, \mathbb{K}) \cong Q_{\Lambda}(2 n-1+d, \mathbb{K})$.

Moreover, $\operatorname{rk}_{\mathrm{emb}}(W(2 n-1, \mathbb{K}))=\operatorname{rk}_{\mathrm{gen}}(W(2 n-1, \mathbb{K}))=2 n+d$.

The following corollary is an immediate consequence of Theorem 1.1.

Corollary 1.2 The isomorphism type of $Q_{\Lambda}(2 n-1+d, \mathbb{K})$ does not depend on the choice of the $\mathbb{K}^{2}$-basis $\Lambda$ of $\mathbb{K}$.

In view of Tits $[5,8.6]$, the embedding rank of $Q(2 n, \mathbb{K})$ is equal to $2 n+1$. Moreover, a quadric isomorphic to $Q(2 n, \mathbb{K})$ arises by intersecting $Q(2 n-1+$ $d, \mathbb{K})$ with the subspace of $\mathrm{PG}(2 n-1+d, \mathbb{K})$ described by the following equations: $x_{2 n+i}=0$ for $0<i<\delta$. Therefore:

Corollary 1.3 We have $W(2 n-1, \mathbb{K}) \cong Q(2 n, \mathbb{K})$ if and only if $d=1$, namely $\mathbb{K}$ is perfect. If $d>1$, then $Q(2 n, \mathbb{K})$ is isomorphic to a proper subspace of $W(2 n-1, \mathbb{K})$.

The error of taking $(\dagger)$ as true even when $\mathbb{K}$ is non-perfect has polluted a nonlittle part of the literature, although not in a ruinous way. A list of all places where that mistake has occurred would certainly tickle the curiosity of many readers, but it would take too long, and perhaps it is not so important. Indeed, once we are aware of that mistake, we can get shelter from it in the following simple way: whenever $(\dagger)$ is used in an apparently essential way, and you are not aware of any alternative argument by which to avoid $(\dagger)$, then assume that $\mathbb{K}$ is perfect. We only mention that the second author of this paper is indeed one of the many that have fallen into that mistake (see [2, page 14, line 15]). 
The rest of this paper is organized as follows. Section 2 contains the proof of Theorem 1.1. As remarked in Corollary 1.3, $Q(2 n, \mathbb{K})$ can be embedded in $W(2 n-1, \mathbb{K})$. In Section 3 we will show that we can also laxly embed $W(2 n-1, \mathbb{K})$ in $Q(2 n, \mathbb{K})$ and that this embedding induces a full embedding of the dual $D W(2 n-1, \mathbb{K})$ of $W(2 n-1, \mathbb{K})$ into the dual $D Q(2 n, \mathbb{K})$ of $Q(2 n, \mathbb{K})$.

\section{Proof of Theorem 1.1}

With $q$ as in $(*)$, let $R$ be the radical of the sesquilinearization of $q$. Then $R$ is defined by the equations $x_{i}=0$ for $i=0,1, \ldots, 2 n-1$. For $0 \leq i<2 n+\delta$, let $u_{i}$ be the vector with all entries equal to 0 but the $i$-th one, which is 1 . Then $U:=\left\{u_{i}\right\}_{0 \leq i<2 n+\delta}$ is a basis of $V(2 n+d, \mathbb{K})$ and $U_{R}:=\left\{u_{i}\right\}_{2 n \leq i<\delta}$ is a basis of $R$. As $\Lambda$ is a $\mathbb{K}^{2}$-basis of $\mathbb{K}, R$ contains no points of the quadric $Q_{\delta}:=Q_{\Lambda}(2 n-1+d, \mathbb{K})$.

Lemma 2.1 Let $X$ be a subspace of $\mathrm{PG}(2 n-1+d, \mathbb{K})$ containing $R$ as a subspace of codimension 1 . Then $X$ contains exactly one point of $Q_{\delta}$.

Proof. Pick a vector $u \in X \backslash R$. Modulo addition of vectors of $R$, we may assume that $u \in\left\langle u_{0}, u_{1}, \ldots, u_{2 n-1}\right\rangle$. Every vector $x \in X$ can be written as $x=\alpha u+r$, for a suitable scalar $\alpha \in \mathbb{K}$ and a vector $r \in R$. Accordingly, $q(x)=\alpha^{2} q(u)+q(r)$. If $q(u)=0$, then $\langle u\rangle$ is the unique point of $Q_{\delta} \cap X$ (indeed $q(r) \neq 0$ for every $r \in R \backslash\{0\})$. Suppose that $q(u) \neq 0$. Then $q(u)$ can be expressed in a unique way as a $\mathbb{K}^{2}$-linear combination of elements of $\Lambda$, say $q(u)=\sum_{j=1}^{k} \alpha_{j} \lambda_{i_{j}}$ where $\alpha_{1}, \alpha_{2}, \ldots, \alpha_{k} \in \mathbb{K}^{2}$. For $j=1,2, . ., k$, put $\beta_{j}:=\sqrt{\alpha_{j}}$ (which exists as $\alpha_{j} \in \mathbb{K}^{2}$ ). Put

$$
v:=u+\sum_{j=1}^{k} \beta_{j} u_{2 n+i_{j}} .
$$

Then $\langle v\rangle$ is the unique point of $Q_{\delta} \cap X$.

By Lemma 2.1, the natural projection of $V(2 n+d, \mathbb{K})$ over $V(2 n+d, \mathbb{K}) / R$ induces an isomorphism from $Q_{\delta}$ to the symplectic polar space $W(2 n-1, \mathbb{K})$ associated with the vector space $V(2 n+d, \mathbb{K}) / R$ on which the sesquilinearization of $q$ defines a nondegenerate alternating form. The first claim of Theorem 1.1 is proved.

By Tits $[5,8.6]$, the embedding of $Q_{\delta}$ in $\mathrm{PG}(2 n-1+d, \mathbb{K})$, implicit in the definition of $Q_{\delta}$, is universal. Hence the isomorphism $W(2 n-1, \mathbb{K}) \cong Q_{\delta}$ also yields the universal embedding of $W(2 n-1, \mathbb{K})$. Therefore $\operatorname{rk}_{\mathrm{emb}}(W(2 n-$ $1, \mathbb{K}))=2 n+d$.

It remains to prove that $\operatorname{rk}_{\text {gen }}(W(2 n-1, \mathbb{K}))=2 n+d$. To this aim, in view of the isomorphism $W(2 n-1, \mathbb{K}) \cong Q_{\delta}$, we only need to construct a generating set of $Q_{\delta}$ of size $2 n+d$.

For every ordinal number $\gamma \leq \delta$, put $V_{\gamma}:=\left\langle u_{i}\right\rangle_{0 \leq i<2 n+\gamma}$ and $Q_{\gamma}:=Q_{\delta} \cap V_{\gamma}$. (In this definition we have replaced $\operatorname{PG}\left(V_{\gamma}\right)$ by $V_{\gamma}$, which is an abuse, but it 
is harmless and makes our notation easier.) Clearly, $V_{\delta}=V(2 n+d$, $\mathbb{K})$. The following are also clear:

(V1) Let $\theta<\gamma \leq \delta$. Then $V_{\theta}$ is a subspace of $V_{\gamma}$.

(V2) Let $\gamma<\delta$. Then $V_{\gamma}$ is a hyperplane of $V_{\gamma+1}$.

(V3) Let $\gamma \leq \delta$ be a limit ordinal. Then $V_{\gamma}=\cup_{\xi<\gamma} V_{\xi}$.

Accordingly:

(Q1) Let $\theta<\gamma \leq \delta$. Then $Q_{\theta}$ is a subspace of $Q_{\gamma}$.

(Q2) Let $\gamma<\delta$. Then $Q_{\gamma}$ is a hyperplane of $Q_{\gamma+1}$.

(Q3) Let $\gamma \leq \delta$ be a limit ordinal. Then $Q_{\gamma}=\cup_{\xi<\gamma} Q_{\xi}$.

Note also that the quadric $Q_{\gamma}$ is non-singular, for every $\gamma \leq \delta$. In particular, $Q_{0} \cong Q^{+}(2 n-1, \mathbb{K})$ and $Q_{1} \cong Q(2 n, \mathbb{K})$. Suppose now that, for a given $\gamma \leq \delta$ we have found a generating set $X_{\xi}$ of $Q_{\xi}$ for every $\xi<\gamma$.

Lemma 2.2 (1) If $\gamma$ is a limit ordinal, then $\cup_{\xi<\gamma} X_{\xi}$ generates $Q_{\gamma}$.

(2) If $\gamma=\theta+1$, let $p$ be a point of $Q_{\gamma} \backslash Q_{\theta}$. Then $X_{\theta} \cup\{p\}$ generates $Q_{\gamma}$.

Proof. Claim (1) immediately follows from (Q3). Turning to (2), suppose that $\gamma=\theta+1$. Then $Q_{\theta}$ is a hyperplane of $Q_{\gamma}$, by (Q2). Moreover, hyperplanes of non-degenerate polar spaces of rank $n>1$ are maximal subspaces. So, given $p \in Q_{\gamma} \backslash Q_{\theta}, Q_{\theta} \cup\{p\}$ generates $Q_{\gamma}$. As $X_{\theta}$ generates $Q_{\theta}, X_{\theta} \cup\{p\}$ generates $Q_{\gamma}$.

We are now ready to construct a generating set $X_{\delta}$ of $Q_{\delta}$ of size $2 n+d$. We define a sequence $\left(X_{\gamma}\right)_{\gamma \leq \delta}$ by transfinite recursion, as follows:

(GS1) $X_{0}$ is a generating set of $Q_{0} \cong Q^{+}(2 n-1, \mathbb{K})$ of size $2 n$. Indeed, it is well known that $Q^{+}(2 n-1, \mathbb{K})$ admits such a generating set. For instance, the set of points of an apartment of $Q^{+}(2 n-1, \mathbb{K})$ is such a set (Cooperstein and Shult [4]; also Blok and Brouwer [1]).

(GS2) If $\gamma=\theta+1$ then we set $X_{\gamma}:=X_{\theta} \cup\left\{p_{\gamma}\right\}$, for a point $p_{\gamma} \in Q_{\gamma} \backslash Q_{\theta}$.

(GS3) If $\gamma \leq \delta$ is a limit ordinal, then $X_{\gamma}:=\cup_{\xi<\gamma} X_{\xi}$.

By Lemma 2.2, (GS1) and transfinite induction, $X_{\gamma}$ generates $Q_{\gamma}$ for every $\gamma \leq \delta$. In particular, $X_{\delta}$ generates $Q_{\delta}$. Moreover, by (GS1) and transfinite induction, $\left|X_{\gamma}\right|=2 n+|\gamma|$ for every $\gamma \leq \delta$ (where $|\gamma|$ stands for the cardinality of $\gamma$. In particular, $\left|X_{\delta}\right|=2 n+d$, as we wanted. 


\section{$3 \quad$ Embedding $Q(2 n, \mathbb{K})$ in $W(2 n-1, \mathbb{K})$ and $W(2 n-$ $1, \mathbb{K})$ in $Q(2 n, \mathbb{K})$}

\subsection{A few definitions}

We have mentioned embeddings in the Introduction, in particular full projective embeddings, implicitly assuming that the reader is familiar with this notion. However that assumption might be partly illegitimate. Indeed, whereas a rich literature exists on projective embeddings, so that we are allowed to assume that readers are aware of them, we also mentioned embeddings between polar spaces and dual polar spaces at the end of the Introduction, but embeddings like these are seldom considered in the literature. We shall now state a quite general definition of embeddings, which comprises the usual definition of projective embeddings of point-line geometries as well as embeddings between polar spaces and dual polar spaces as understood in our Introduction.

We recall that a point-line geometry is a pair $\mathcal{G}=(P, L)$, where $P$ (the set of points) is a non-empty set and $L$ is a family of subsets of $P$, called lines, such that no two lines have more than one point in common and every line has at least two points. The collinearity graph of $\mathcal{G}$ is the graph with $P$ as the set of vertices and 'being collinear' as the adjacency relation. A subspace of $\mathcal{G}$ is a subset $X \subseteq P$ such that, for every line $l \in L$, if $|l \cap X|>1$ then $l \subseteq X$. Clearly, $P$ is a subspace of $\mathcal{G}$ (the improper one) and the intersection of any family of subspaces of $\mathcal{G}$ is a subspace of $\mathcal{G}$. Given a subset $X \subseteq P$, the subspace of $\mathcal{G}$ spanned by $X$ (also called the span of $X$ ) is the smallest subspace of $\mathcal{G}$ containing $X$, namely the intersection of all subspaces of $\mathcal{G}$ containing $X$.

Let $\mathcal{G}_{1}=\left(P_{1}, L_{1}\right)$ and $\mathcal{G}_{2}=\left(P_{2}, L_{2}\right)$ be two point-line geometries. An embedding $e: \mathcal{G}_{1} \rightarrow \mathcal{G}_{2}$ is an injective mapping $e: P_{1} \rightarrow P_{2}$ satisfying both the followings:

- (E1) for every line $l \in L_{1}$, the set $e(l):=\{e(x)\}_{x \in l}$ spans a line of $\mathcal{G}_{2}$;

- (E2) different lines of $\mathcal{G}_{1}$ are mapped by $e$ into different lines of $\mathcal{G}_{2}$.

If moreover $e(l)$ is a line of $\mathcal{G}_{2}$ for every line $l \in L_{1}$, then we say that the embedding $e$ is full. Non-full embeddings will be called lax.

If the set $e\left(P_{1}\right)$ spans a proper subspace of $\mathcal{G}_{2}$ then we say that the embedding $e$ is loose, otherwise $e$ is said to be strict. According to this terminology, a projective embedding of $\mathcal{G}_{1}$ is a strict embedding $e: \mathcal{G}_{1} \rightarrow \mathcal{G}_{2}$ where $\mathcal{G}_{2}$ is a projective space.

Turning back to the general case, given an embedding $e: \mathcal{G}_{1} \rightarrow \mathcal{G}_{2}$ and denoted by $d_{i}(.,$.$) the distance function in the collinearity graph of \mathcal{G}_{i}$ for $i=$ 1,2 , we have $d_{2}(e(x), e(y)) \leq d_{1}(x, y)$ for any two points $x, y \in P_{1}$. When $d_{2}(e(x), e(y))=d_{1}(x, y)$ for any two points $x, y \in P_{1}$ then we say that $e$ is isometric.

We finish this subsection by stating an easy lemma, the proof of which is left to the reader. 
Lemma 3.1 Given two point-line geometries $\mathcal{G}_{1}=\left(P_{1}, L_{1}\right)$ and $\mathcal{G}_{2}=\left(P_{2}, L_{2}\right)$, let $e: \mathcal{G}_{1} \rightarrow \mathcal{G}_{2}$ be an embedding. Assume the following:

(1) the embedding e is full;

(2) for any two points $x, y \in P_{1}$, if $e(x)$ and $e(y)$ are collinear in $\mathcal{G}_{2}$ then $x$ and $y$ are collinear in $\mathcal{G}_{1}$.

Then $e\left(P_{1}\right)$ is a subspace of $\mathcal{G}_{2}$ and $e$ induces an isomorphism from $\mathcal{G}_{1}$ to the geometry induced by $\mathcal{G}_{2}$ on $e\left(P_{1}\right)$. In particular, if $e$ is also strict then $e$ is an isomorphism from $\mathcal{G}_{1}$ to $\mathcal{G}_{2}$.

\subsection{Embedding $Q(2 n, \mathbb{K})$ in $W(2 n-1, \mathbb{K})$}

The quadric $Q(2 n, \mathbb{K})$ can be fully embedded in $W(2 n-1, \mathbb{K})$. We have already noticed this fact in Corollary 1.3, but we want to examine it a bit closer.

With $\delta$ and $d$ as in the Introduction, suppose $d>1$ and let $J$ be a nonempty proper subset of $\{i\}_{0 \leq i<\delta}$. Put $\bar{J}=\{0,1, \ldots, 2 n-1\} \cup\{2 n+i \mid i \in J\}$ and

$$
q_{J}\left(\left(x_{i}\right)_{i \in \bar{J}}\right):=\sum_{i=0}^{n-1} x_{i} x_{n+i}+\sum_{i \in J} \lambda_{i} x_{2 n+i}^{2} .
$$

Let $Q_{J}(\mathbb{K})$ be the quadric associated to $q_{J}$. In particular, if $|J|=1$ then $Q_{J}(\mathbb{K}) \cong Q(2 n, \mathbb{K})$.

We may regard the form $q_{J}$ as defined over the subspace $S_{J}$ of $V:=V(2 n+$ $d, \mathbb{K})$ defined by the equations $x_{2 n+i}=0$ for $i \notin J$. The inclusion $S_{J} \subset V$ naturally defines an embedding $e_{J}: Q_{J}(\mathbb{K}) \rightarrow Q_{\Lambda}(2 n-1+d, \mathbb{K})$ as well as an embedding $e_{J}^{*}$ of the dual $D Q_{J}(\mathbb{K})$ of $Q_{J}(\mathbb{K})$ into the dual $D Q_{\Lambda}(2 n-1+d, \mathbb{K})$ of $Q_{\Lambda}(2 n-1+d, \mathbb{K})$. In view of the isomorphism $W(2 n-1, \mathbb{K}) \cong Q_{\Lambda}(2 n-1+d, \mathbb{K})$, we may also regard $e_{J}$ and $e_{J}^{*}$ as embeddings of $Q_{J}(\mathbb{K})$ into $W(2 n-1, \mathbb{K})$ and of $D Q_{J}(\mathbb{K})$ into the dual $D W(2 n-1, \mathbb{K})$ of $W(2 n-1, \mathbb{K})$ respectively. The following is clear:

Theorem 3.2 The embedding $e_{J}$ is full and isometric but loose.

The rest of this subsection is devoted to the investigation of $e_{J}^{*}$.

Lemma 3.3 The embedding $e_{J}^{*}$ is non-full.

Proof. Let $u_{1}, u_{2}, \ldots, u_{n-1}, v, w$ be vectors of $S_{J}$ such that $\left\langle u_{1}, u_{2}, \ldots, u_{n-1}, v\right\rangle$ and $\left\langle u_{1}, u_{2}, \ldots, u_{n-1}, w\right\rangle$ are maximal singular subspaces of $Q_{J}(\mathbb{K})$. We only need to prove that there exists a vector $u_{0} \in V \backslash S_{J}$ and scalars $x, y \in \mathbb{K}$ such that $x v+v w+u_{0}$ is singular and orthogonal to $\left\{u_{1}, u_{2}, \ldots, u_{n-1}\right\}$.

Denoted by $f$ the sesquilinearization of $q_{\Lambda}$, we may assume to have chosen $v$ and $w$ in such a way that $f(v, w)=1$. Given $0 \leq j<\delta$ with $j \notin J$, let $u_{0}$ be the vector of $V$ with the $(2 n+j)$ th entry equal to 1 and all remaining entries equal to 0 . Then $\lambda_{j} v+w+u_{0}$ is singular and orthogonal to $\left\{u_{1}, u_{2}, \ldots, u_{n-1}\right\}$, but $\left\langle u_{1}, u_{2}, \ldots, u_{n-1}, \lambda_{j} v+w+u_{0}\right\rangle$ is not a singular subspace of $Q_{J}(\mathbb{K})$.

Lemma 3.4 The embedding $e_{J}^{*}$ is isometric. 
Proof. Denoted by $d_{J}$ and $d_{\Lambda}$ the distance functions in $D Q_{J}(\mathbb{K})$ and $D Q_{\Lambda}(2 n-$ $1+d, \mathbb{K})$ respectively, for two maximal singular subspaces $X, Y$ of $Q_{J}(\mathbb{K})$ we have $d_{J}(X, Y)=\operatorname{cod}_{X}(X \cap Y)$ and $d_{\Lambda}\left(e_{J}(X), e_{J}(Y)\right)=\operatorname{cod}_{e_{J}(X)}\left(e_{J}(X) \cap e_{J}(Y)\right)$. However, it is clear that $\operatorname{cod}_{X}(X \cap Y)=\operatorname{cod}_{e_{J}(X)}\left(e_{J}(X) \cap e_{J}(Y)\right)$. Hence $e_{J}^{*}$ is isometric.

Henceforth $\mathcal{M}$ denotes the set of maximal singular subspaces of $Q_{J}(\mathbb{K})$, namely the set of points of $D Q_{J}(\mathbb{K})$. We recall that, given a graph $\Gamma=(P, \sim)$, a subset $S \subseteq P$ is said to be convex if, for any two vertices $x, y \in S$, all shortest paths of $\Gamma$ between $x$ and $y$ are contained in $S$.

Lemma 3.5 The set $e_{J}^{*}(\mathcal{M})$ is a convex set of vertices of the collinearity graph of $D Q_{\Lambda}(2 n-1+d, \mathbb{K})$.

Proof. Given $X, Y \in e_{J}^{*}(\mathcal{M})$, let $X_{0}=X, X_{1}, \ldots, X_{k-1}, X_{k}=Y$ be a shortest path of $D Q_{\Lambda}(2 n-1+d, \mathbb{K})$ from $X$ to $Y$. We shall prove that all points $X_{1}, X_{2}, \ldots, X_{k-1}$ belong to $e_{J}^{*}(\mathcal{M})$. We shall prove this fact by induction on $k$. If $k=1$ there is nothing to prove. Let $k>1$, let $\bar{X}, \bar{Y} \in \mathcal{M}$ be such that $e_{J}^{*}(\bar{X})=X$ and $e_{J}^{*}(\bar{Y})=Y$ and let $l$ be the line of $D Q_{\Lambda}(2 n-1+d, \mathbb{K})$ through $X_{k-1}$ and $X_{k}=Y$. The lines of $D Q_{\Lambda}(2 n-1+d, \mathbb{K})$ through $Y$ (the lines of $D Q_{J}(\mathbb{K})$ through $\left.\bar{Y}\right)$ bijectively correspond to the hyperplanes of the $(n-1)$ dimensional projective space $Y$ (respectively $\bar{Y}$ ) and $e_{J}$ induces an isomorphism from the projective space $\bar{Y}$ to the projective space $Y$. Therefore there exists a line $\bar{l}$ of $D Q_{J}(\mathbb{K})$ through $\bar{Y}$ that is mapped into $l$ by $e_{J}^{*}$. Let $\bar{Z}$ be the point of $\bar{l}$ closest to $\bar{X}$. As $e_{J}^{*}$ is isometric and maps $\bar{l}$ into $l$, the point $Z=e_{J}^{*}(\bar{Z})$ is the point of $l$ closest to $X$. Hence $Z=X_{k-1}$. Therefore $X_{k-1} \in e_{J}^{*}(\mathcal{M})$. We can now apply the inductive hypothesis on $k$ to the pair $\left\{X, X_{k-1}\right\}$, thus obtaining that all points $X_{1}, X_{2}, \ldots, X_{k-1}$ belong to $e_{J}^{*}(\mathcal{M})$.

In view of the sequel, we need a general lemma on dual polar spaces.

Lemma 3.6 Given a thick dual polar space $\Delta$, let $S$ be a convex set of vertices of the collinearity graph of $\Delta$ containing at least one pair of points at maximal distance in $\Delta$. Then $S$ generates $\Delta$.

Proof. We shall argue by induction on the diameter $d$ of $\Delta$. If $d=1$ there is nothing to prove. Suppose $d>1$ and let $x, y$ be points of $S$ at distance $d$. For every point $z$ of $\Delta$ at distance $d_{1}<d$ from $x$, let $Z$ be the convex closure of $\{x, z\}$ in $\Delta$ and $y_{Z}$ the projection of $y$ onto $Z$. Then $y_{Z}$ belongs to a shortest path of $\Delta$ from $y$ to $x$. Hence $y_{Z} \in S$, as $S$ is convex. Moreover, $y$ has distance $d-d_{1}$ from $Z$. Hence the distance between $x$ and $y_{Z}$ is equal to $d_{1}$. On the other hand, $Z$ is a dual polar space of diameter $d_{1}$. We can apply our inductive hypothesis to $Z \cap S$, thus obtaining that $z$ belongs to the span of $Z \cap S$ in $Z$. Therefore the span of $S$ in $\Delta$ contains the set $H_{x}$ of all points of $\Delta$ at nonmaximal distance from $x$. This set is a hyperplane of $\Delta$, whence a maximal subspace of $\Delta$ (see Brouwer and Wilbrink [3]). However, $S$ also contains $y$, 
which does not belong to $H_{x}$. Therefore the span of $S$ contains all points of $\Delta$.

We can now prove the main result of this subsection.

Theorem 3.7 The embedding $e_{J}^{*}$ is strict and isometric but non-full.

Proof. We already know that $e_{J}^{*}$ is isometric and non-full (Lemmas 3.3 and 3.4). It remains to prove that $e_{J}^{*}(\mathcal{M})$ spans the whole point-set of $D Q_{\Lambda}(2 n-1+d, \mathbb{K})$. By Lemma 3.5, the set $e_{J}^{*}(\mathcal{M})$ is convex. Moreover, since $e_{J}^{*}$ is isometric and both $D Q_{J}(\mathbb{K})$ and $D Q_{\Lambda}(2 n-1+d, \mathbb{K})$ have diameter equal to $n$, the set $e_{J}^{*}(\mathcal{M})$ contains pairs of points of $D Q_{\Lambda}(2 n-1+d, \mathbb{K})$ at distance $n$. By Lemma 3.6, that set spans $D Q_{\Lambda}(2 n-1+d, \mathbb{K})$.

\subsection{Embedding $W(2 n-1, \mathbb{K})$ in $Q(2 n, \mathbb{K})$}

Again, we will suppose that $d>1$. Let $\sqrt{\mathbb{K}}$ be the extension of $\mathbb{K}$ obtained by adding a square-root of $k$ for all $k \in \mathbb{K}$. Clearly, $\sqrt{\mathbb{K}}$ is contained in the quadratic closure of $\mathbb{K}$. Since $d>1, \sqrt{\mathbb{K}} \neq \mathbb{K}$.

We shall firstly show that $W(2 n-1, \mathbb{K})$ admits a lax embedding in $Q(2 n, \sqrt{\mathbb{K}})$. In view of Theorem 1.1, we only need to exhibit a lax embedding of $Q_{\Lambda}(2 n-$ $1+d, \mathbb{K})$ in $Q(2 n, \sqrt{\mathbb{K}})$. This can be done as follows. For every $i<\delta$, let $\sqrt{\lambda_{i}}$ be the square root of $\lambda_{i}$ in $\sqrt{\mathbb{K}}$. Define $\theta: V(2 n+d, \mathbb{K}) \rightarrow V(2 n+1, \sqrt{\mathbb{K}})$ as follows: $\theta\left(\left(x_{i}\right)_{0 \leq i<2 n+\delta}\right)=\left(x_{0}, x_{1}, \ldots, x_{2 n-1}, z\right)$ where

$$
\text { (1) } z:=\sum_{0 \leq i<\delta}\left(\sqrt{\lambda_{i}}\right) x_{2 n+i} .
$$

Note that the mapping $\theta$ defined in this way is $\mathbb{K}$-linear. We may also assume that $Q(2 n, \sqrt{\mathbb{K}})$ is defined by the following quadratic form:

$$
\text { (2) } q\left(x_{0}, x_{1}, \ldots, x_{2 n-1}, z\right):=\sum_{i=0}^{2 n-1} x_{i} x_{n+i}+z^{2} .
$$

Lemma 3.8 (i) $\theta$ induces a lax embedding $e_{\theta}: Q_{\Lambda}(2 n-1+d, \mathbb{K}) \rightarrow Q(2 n, \sqrt{\mathbb{K}})$ as well as an embedding $e_{\theta}^{*}: D Q_{\Lambda}(2 n-1+d, \mathbb{K}) \rightarrow D Q(2 n, \sqrt{\mathbb{K}})$.

(ii) Both $e_{\theta}$ and $e_{\theta}^{*}$ are isometric.

Proof. As we have mentioned in Section 2, the map $\theta_{1}: V(2 n+d, \mathbb{K}) \rightarrow$ $V(2 n, \mathbb{K}),\left(x_{i}\right)_{0 \leq i<2 n+\delta} \mapsto\left(x_{0}, x_{1}, \ldots, x_{2 n-1}\right)$ defines an isomorphism between the polar spaces $Q_{\Lambda}(2 n-1+d, \mathbb{K})$ and $W(2 n-1, \mathbb{K})$ and hence preserves the dimensions of the (intersection of) singular subspaces. Since every line of $\mathrm{PG}(2 n, \sqrt{\mathbb{K}})$ through the nucleus of $Q(2 n, \sqrt{\mathbb{K}})$ contains at most 1 point of $Q(2 n, \sqrt{\mathbb{K}})$, the map $\theta_{2}: V(2 n+1, \sqrt{\mathbb{K}}) \rightarrow V(2 n, \sqrt{\mathbb{K}}),\left(x_{0}, x_{1}, \ldots, x_{2 n-1}, z\right) \mapsto$ $\left(x_{0}, x_{1}, \ldots, x_{2 n-1}\right)$ defines a map from the set of singular subspaces of $Q(2 n, \sqrt{\mathbb{K}})$ to the set of subspaces of $\mathrm{PG}(2 n-1, \sqrt{\mathbb{K}})$ preserving the dimensions of the (intersection of) subspaces. The natural inclusion $\theta_{3}$ of $V(2 n, \mathbb{K})$ in $V(2 n, \sqrt{\mathbb{K}})$ 
defines a map from the set of subspaces of $\mathrm{PG}(2 n-1, \mathbb{K})$ to the set of subspaces of $\mathrm{PG}(2 n-1, \sqrt{\mathbb{K}})$ preserving the dimensions of the (intersection of) subspaces.

Since $\theta_{2} \theta=\theta_{3} \theta_{1}, \theta$ defines a map from the set of singular subspaces of $Q_{\Lambda}(2 n-1+d, \mathbb{K})$ to the set of singular subspaces of $Q(2 n, \sqrt{\mathbb{K}})$ preserving the dimensions of the (intersection of) subspaces. The claims of the lemma are now easily verified.

Lemma 3.9 The embedding $e_{\theta}^{*}$ is full.

Proof. Let $u_{1}, u_{2}, \ldots, u_{n-1}, v, w$ be vectors of $V=V(2 n+d, \mathbb{K})$ such that $\left\langle u_{1}, u_{2}, \ldots, u_{n-1}, v\right\rangle$ and $\left\langle u_{1}, u_{2}, \ldots, u_{n-1}, w\right\rangle$ are maximal singular subspaces of $Q_{\Lambda}(2 n-1+d, \mathbb{K})$. We may assume to have chosen $v, w$ in such a way that $f(v, w)=1$, where $f$ is the sesquilinearization of the form $q_{\Lambda}$. Let $\bar{u}$ be the vector of $V(2 n+1, \sqrt{\mathbb{K}})$ with the last entry equal to 1 and all remaining coordinates equal to 0 . Namely $\bar{u}$ is a representative of the nucleus of $Q(2 n, \sqrt{\mathbb{K}})$. Let $S$ be a maximal singular subspace of $Q(2 n, \sqrt{\mathbb{K}})$ containing $\left\langle\theta\left(u_{1}\right), \ldots, \theta\left(u_{n-1}\right)\right\rangle$. Then $S$ is spanned by $\theta\left(u_{1}\right), \theta\left(u_{2}\right), \ldots, \theta\left(u_{n-1}\right)$ and a vector $x \theta(v)+y \theta(w)+z \bar{u}$ for suitable scalars $x, y, z \in \sqrt{\mathbb{K}}$ such that $x y=z^{2}$. Indeed $f(v, w)=1$ by assumption and, if $g$ is the sesquilinearization of the quadratic form $q$ giving rise to $Q(2 n, \sqrt{\mathbb{K}})$, then $g(\theta(v), \theta(w))=f(v, w)$.

As $x y=z^{2}$ and not all of $x, y, z$ are zero, we have $x \neq 0$ or $y \neq 0$. Without loss of generality, we may suppose that $y \neq 0$. Modulo re-scaling the vector $x \theta(v)+y \theta(w)+z \bar{u}$, we may suppose that $y=1$. So, $x=x y=z^{2} \in \mathbb{K}$ and $S=\left\langle\theta\left(u_{1}\right), \ldots, \theta\left(u_{n-1}\right), \theta(x v)+\theta(w)+z \bar{u}\right\rangle$. It suffices to show that the vector $\theta(x v)+\theta(w)+z \bar{u}$ belongs to the image of $\theta$. As $\theta$ is $\mathbb{K}$-linear, we only need to show that $z \bar{u}$ belongs to the image of $\theta$. Now, we can write $z^{2}$ in the form $\sum_{j=1}^{k} \alpha_{j} \lambda_{i_{j}}$, where $\alpha_{1}, \ldots, \alpha_{k} \in \mathbb{K}^{2}$. Then $z \bar{u}=\theta\left(\left(x_{i}\right)_{0 \leq i<2 n+\delta}\right)$, where $x_{i}$ is equal to $\sqrt{\alpha_{j}}$ if $i=2 n+i_{j}(j \in\{1, \ldots, k\})$ and equal to 0 otherwise.

As $e_{\theta}^{*}$ is isometric and full (Lemmas 3.8 and 3.9), Lemma 3.1 implies that the image $e_{\theta}^{*}\left(D Q_{\Lambda}(2 n-1+d, \mathbb{K})\right)$ of $D Q_{\Lambda}(2 n-1+d, \mathbb{K})$ by $e_{\theta}^{*}$ is a subspace of $D Q(2 n, \sqrt{\mathbb{K}})$ and $e_{\theta}^{*}$ induces an isomorphism from $D Q_{\Lambda}(2 n-1+d, \mathbb{K})$ to the geometry induced by $D Q(2 n, \sqrt{\mathbb{K}})$ on $e_{\theta}^{*}\left(D Q_{\Lambda}(2 n-1+d, \mathbb{K})\right)$. However, $Q_{\Lambda}(2 n-1+d, \mathbb{K}) \neq Q(2 n, \sqrt{\mathbb{K}})$. (This immediately follows from Tits [5, 8.6].) Hence $D Q_{\Lambda}(2 n-1+d, \mathbb{K}) \neq D Q(2 n, \sqrt{\mathbb{K}})$. Therefore $e_{\theta}^{*}\left(D Q_{\Lambda}(2 n-1+d, \mathbb{K})\right)$ is a proper subspace of $D Q(2 n, \sqrt{\mathbb{K}})$. Thus, $e_{\theta}^{*}$ is loose. On the other hand:

Lemma 3.10 The embedding $e_{\theta}$ is strict.

Proof. Let $Q^{+}(2 n-1, \mathbb{K})$ be the quadric obtained by intersecting $Q_{\Lambda}(2 n-1+$ $d, \mathbb{K})$ with the subspace determined by the equations $x_{2 n+i}=0(0 \leq i<\delta)$, and let $Q^{+}(2 n-1, \sqrt{\mathbb{K}})$ be the quadric obtained by intersecting $Q(2 n, \sqrt{\mathbb{K}})$ with the subspace $z=0$. Then $e_{\theta}\left(Q^{+}(2 n-1, \mathbb{K})\right) \subseteq Q^{+}(2 n-1, \sqrt{\mathbb{K}})$ and every apartment of $Q^{+}(2 n-1, \mathbb{K})$ is mapped by $e_{\theta}$ to an apartment of $Q^{+}(2 n-1, \sqrt{\mathbb{K}})$. Since $Q^{+}(2 n-1, \sqrt{\mathbb{K}})$ is spanned by any of its apartments (recall [1] and [4]), we have that $e_{\theta}\left(Q^{+}(2 n-1, \mathbb{K})\right)$ spans $Q^{+}(2 n-1, \sqrt{\mathbb{K}})$. If $p$ is a point of 
$Q_{\Lambda}(2 n-1+d, \mathbb{K}) \backslash Q^{+}(2 n-1, \mathbb{K})$, then $e_{\theta}(p) \in Q(2 n, \sqrt{\mathbb{K}}) \backslash Q^{+}(2 n-1, \sqrt{\mathbb{K}})$ and hence $Q(2 n, \sqrt{\mathbb{K}})$ is spanned by $Q^{+}(2 n-1, \sqrt{\mathbb{K}}) \cup\left\{e_{\theta}(p)\right\}$. It follows that $Q(2 n, \sqrt{\mathbb{K}})$ is spanned by $e_{\theta}\left(Q_{\Lambda}(2 n-1+d, \mathbb{K})\right)$.

So far, we have proved the following.

Theorem 3.11 The embedding $e_{\theta}$ is isometric and strict but non-full. The embedding $e_{\theta}^{*}$ is full and isometric, but loose.

Let now $\sigma$ be the semi-linear endomorphism of $V(2 n+1, \sqrt{\mathbb{K}})$ sending every vector $\left(y_{i}\right)_{i=0}^{2 n} \in V(2 n+1, \sqrt{\mathbb{K}})$ to $\left(y_{i}^{2}\right)_{i=0}^{2 n}$. Then $\sigma$ induces an isomorphism between the projective geometries $\mathrm{PG}(2 n, \sqrt{\mathbb{K}})$ and $\mathrm{PG}(2 n, \mathbb{K})$. Accordingly, $\sigma$ also defines an isomorphism $e_{\sigma}: Q(2 n, \sqrt{\mathbb{K}}) \rightarrow Q(2 n, \mathbb{K})$ and an isomorphism $e_{\sigma}^{*}: D Q(2 n, \sqrt{\mathbb{K}}) \rightarrow D Q(2 n, \mathbb{K})$.

Put $e:=e_{\sigma} e_{\theta}$ and $e^{*}:=e_{\sigma}^{*} e_{\theta}^{*}$. Regarding $e_{\theta}$ and $e_{\theta}^{*}$ as embeddings of $W(2 n-1, \mathbb{K})$ in $Q(2 n, \sqrt{\mathbb{K}})$ and of $D W(2 n-1, \mathbb{K})$ in $D Q(2 n, \sqrt{\mathbb{K}})$ respectively, as we may by Theorem 1.1 , we obtain embeddings $e: W(2 n-1, \mathbb{K}) \rightarrow Q(2 n, \mathbb{K})$ and $e^{*}: D W(2 n-1, \mathbb{K}) \rightarrow D Q(2 n, \mathbb{K})$. Theorem 3.11 implies the following:

Theorem 3.12 The embedding e : $W(2 n-1, \mathbb{K}) \rightarrow Q(2 n, \mathbb{K})$ is strict and isometric but non-full. The embedding $e^{*}: D W(2 n-1, \mathbb{K}) \rightarrow D Q(2 n, \mathbb{K})$ is full and isometric but loose.

The existence of the embeddings constructed in this subsection and in the previous one evidently has some consequences for the investigation of projective embeddings of $W(2 n-1, \mathbb{K}), Q(2 n, \mathbb{K}), D W(2 n-1, \mathbb{K})$ and $D Q(2 n, \mathbb{K})$, but such an investigation goes beyond the scope we have set for this paper.

\section{References}

[1] R. J. Blok and A. E. Brouwer, Spanning point-line geometries in buildings of spherical type, J. Geometry 62 (1998), 26-35.

[2] R. J. Blok and A. Pasini, Point-line geometries with a generating set that depends on the underlying field, in "Finite Geometries" (eds. J.W.P. Hirschfeld et al.), Kluwer, Dordrecht (2001), 1-25.

[3] A. E. Brouwer and H. A. Wilbrink, The structure of near polygons with quads, Geom. Dedicata 14 (1983), 145-176.

[4] B. N. Cooperstein and E. E. Shult, Frames and bases of Lie incidence geometries, J. Geometry 60 (1997), 17-46.

[5] J. Tits, "Buildings of Spherical Type and Finite BN-pairs", Lecture Notes in Math. 386, Springer, 1974.

[6] H. Van Maldeghem, "Generalized Polygons", Birkhäuser, Basel, 1998. 


\section{Addresses:}

Bart De Bruyn

Department of Pure Mathematics and Computer Algebra

Ghent University

Krijgslaan 281 (S22)

B-9000 Gent, Belgium

bdb@cage.ugent.be

\section{Antonio Pasini}

Dipartimento di Scienze Matematiche e Informatiche

Università di Siena

Pian dei Mantellini, 44

I-53100 Siena, Italy

pasini@unisi.it 